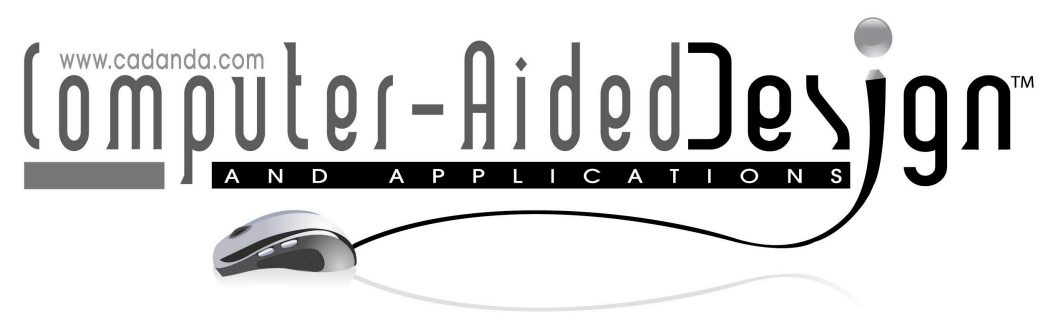

\title{
Using 3D CAD models for value visualization: an approach with SIEMENS NX HD3D Visual Reporting
}

\author{
Marco Bertoni ${ }^{1,2}$, Alessandro Bertoni ${ }^{2}$ Henk Broeze $^{3}$, Gilles Dubourg ${ }^{4}$ Clive Sandhurst $^{5}$ \\ ${ }^{1}$ Blekinge Institute of Technology, marco.bertoni@bth.se \\ ${ }^{2}$ Luleå University of Technology, alessandro.bertoni@ltu.se \\ ${ }^{3}$ Siemens PLM Software, henk.broeze@siemens.com \\ ${ }^{4}$ Siemens PLM Software, gilles.dubourg@siemens.com \\ ${ }^{5}$ Siemens PLM Software, clive.sandhurst@siemens.com
}

\begin{abstract}
Recent literature in Systems Engineering has suggested the use of "value" to drive decision-making activities during preliminary design, in particular when choosing technologies and components for a complex system. However, to correctly evaluate design trade-offs, a visual link has to be established between the results of the value model and a product shape/geometry. This paper proposes the use of color-coded 3D CAD models to support the visualization of value analysis results in a Stage-Gate ${ }^{\circledR}$ process. The approach has been developed and exemplified within a case study related to the design of an aero-engine component, and has been demonstrated using SIEMENS NX HD3D Visual Reporting. The results of verification activities conducted in a laboratory setting show that the use of color-coded 3D CAD models increases the decision makers' awareness of value-related information in a Stage-Gate process.
\end{abstract}

Keywords: value driven design, value visualization, color-coding.

DOI: 10.3722/cadaps.2013.xxx-yyy

\section{INTRODUCTION}

All designs are created for a purpose. When dealing with well-defined and known problems, this purpose is well mirrored by the product requirements, which provide a good enough basis to identify the best of the available design alternatives. However, in long and complex new development processes that involve several supply chain partners, the purpose is often lost when requirements are cascaded down to suppliers and sub-contractors [16]. This causes component manufacturers to develop local optimal solutions that minimize cost, rather than to target innovative technologies that might add value to customers' and stakeholders' processes. In this context, measuring requirements fulfilment is no longer sufficient to assess the "goodness" of a design [7][13], rather more qualitative criteria need to be considered to better understand the value of a solution from a system viewpoint [37].

Recent literature in Systems Engineering [24] and Value Driven Design (VDD) [12] has promoted the use of "value" as a driver for decision-making activities in preliminary design. The ambition is to use "value" to provide a measurable approximation of the level of fulfillment of the overall system needs ensured by a design solution [8]. In practice, so called "value models" are built to quantitatively 
assess how a sub-system will affect the behavior of a system along its lifecycle. From the standpoint of a supply chain partner, understanding how limited design changes at component level impact the system value increases the advantage against competitors, and the ability to negotiate system-level design with the original equipment manufacturer [16].

Creating an environment where engineers and designers can visually link "value" to product components is a necessary step to enable more value-oriented decisions in design [13]. However, the integration of innovative information visualization approaches in daily work practices is a laborintensive and risky process [32]. Large companies require upfront authorization to deploy new software or tools in their working environment, for both functionality and security reasons. Furthermore, experts are often accustomed to and effective with the existing tools and methods, and the integration of a new solution may break the chain of analysis processes [32].

A recent stream of literature [e.g.: 23] promotes the use of CAD/PLM tools for lifecycle information visualization, as a way to limit users' reluctance against new systems. In spite of the shortcomings in conveying usage, manufacturing and service information [22], the recent market trends show that the scope of CAD/PLM is extending to support a wider range of analysis and data, from different fields [38]. Recent releases embed modules and functions aiming at capturing customer needs and technical requirements, defining systems architecture, modeling and validating systems behavior, and managing embedded software [18][35]. CAD models are popular not only because they are easily shareable over the Internet, increasing communication between customers and suppliers [16], but also because they represent a good trade-off between perception of product representation and frequency of use, in comparison with hand-made sketches, scale models, prototypes, mock-ups, construction design virtual reality and rapid prototyping [20].

Emerging from the above considerations, the authors have investigated the use of color-coded 3D CAD models to support visualization of value-related information in the early stages of design. The paper describes how these models have been used to translate the results of a value analysis into visual features in a CAD/PLM environment. These findings emerge from a study conducted within an EU FP7 research project named CRESCENDO [16], which gave the authors access to several European aerospace manufacturing companies and IT vendors.

Firstly, the paper presents the high-level process for value assessment, as defined during the empirical study, to set the context from which preferences for value visualization are extracted and interpreted. Furthermore, it describes how these preferences are translated into an approach for value visualization, which was demonstrated using SIEMENS NX HD3D Visual Reporting capabilities within a case study related to the design of an innovative aero-engine component. Eventually, the paper presents the results of verification activities conducted both with design experts within the case company, as well as with undergraduate students in ad-hoc design sessions.

\section{A CASE STUDY FROM THE AEROSPACE INDUSTRY}

Early in the aircraft and engine design process, the design team is required to reason upon how to improve hardware, software and services to provide a more comfortable, timely, and entertaining flight experience [1][9]. A key area of investigation relates, for instance, to the integration of energy sources (electrical, hydraulic, etc.) to improve the performances of the powerplant. On a more technical level, this demands for altered functions in the engines to improve the efficiency in energy use [30], which turns into new requirements for sub-systems and components [8]. Component manufacturers can address the energy saving target by pursuing different strategies, playing with weight, cost, performance, and functionality. Yet, it is cumbersome to assess in an early stage how a given strategy will contribute to add value to the overall system along its lifecycle, as highlighted by one of authors' contact:

"Nowadays you can easily tell why a solution is the optimal one in terms of performances, however it is not straightforward to see if it is optimal also from a value perspective. Hence, we have to look at people, tools, processes for developing the optimal solution both from a business as well as customer viewpoint".

This issue is particularly evident when designing an innovative aero-engine intermediate compressor case (IMC). The IMC is the biggest static component in an aero-engine and plays a key role 
from both a structural and functional perspective. It includes a core hub, support for thrust lugs, integrated structural fan outlet guide vanes, an outer ring and the engine mounts (Fig. 1). The development of a new technology for bleed air off-take, for instance, raises concerns about performances, weight, maintainability and value of the entire aero-engine system.

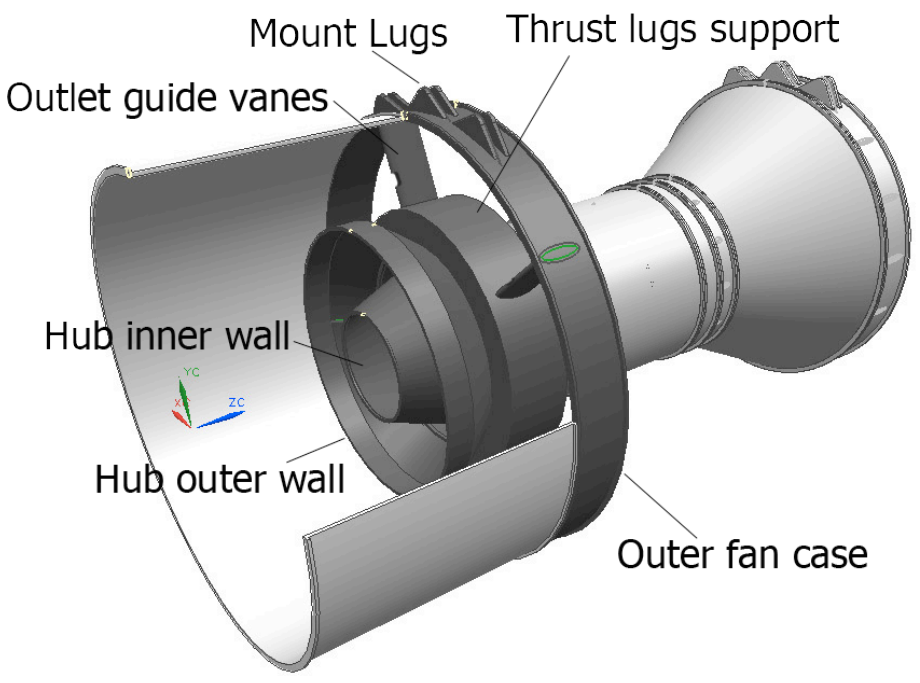

Fig. 1: Position of the IMC (and its main parts) in the aero-engine.

In the studied case, 2 alternative IMC technology concepts were developed, refined and benchmarked from a value perspective. Option \#1 embodies a traditional fully casted design, which implements a bleed off-take function in the core hub. Option \#2 is more radical, featuring an increased use of composite material, but where a bleed air off-take function is not implemented.

\subsection{The Value Assessment Process}

Understanding how the value assessment process is executed during early-stage sub-system design is crucial to determine the main features of the value visualization approach. For the IMC (and other similar components) this is shaped on Stage-Gate ${ }^{\circledR}[14]$, a popular process to guide development projects from idea generation to product launch. The key components of Stage-Gate ${ }^{\circledR}$ are the Stages, where information-gathering activities (summarized by deliverables) take place, and the Gates, where information is assessed and decisions are made. Previous work [8] shows that the awareness on valuerelated information has to be raised both at the Gate, to correctly judge the design trade-offs, and during the Stage, to guide creative processes towards more value-adding solutions (Fig. 2).

At sub-system level, the value assessment process kicks off with the negotiation of relevant value dimensions and drivers against which to benchmark alternatives solution concepts (Phase 1). Value dimensions, which capture major aerospace project needs, are specified into several value drivers, which are more product-specific and directly related to the component under development. For instance, given a dimension such as Profitability, the team might define Machine commonality and Availability as relevant drivers. This phase also concerns the definition of specific objectives for each design. These objectives cascade down the value drivers and describe, with more detail, the behavior of a technological option. For instance, Machine commonality can be translated to \% of reuse of existing turning machines, while Availability can both refer to Mean Time Between Maintenance (MTBM) and Mean Time Between Failure (MTBF). It has to be noted that alternative concepts, although differing in terms of materials, geometry and shape, are rarely completely new products. Rather, they are often variants of an existing technology platform, and are composed by the same basic building blocks. 


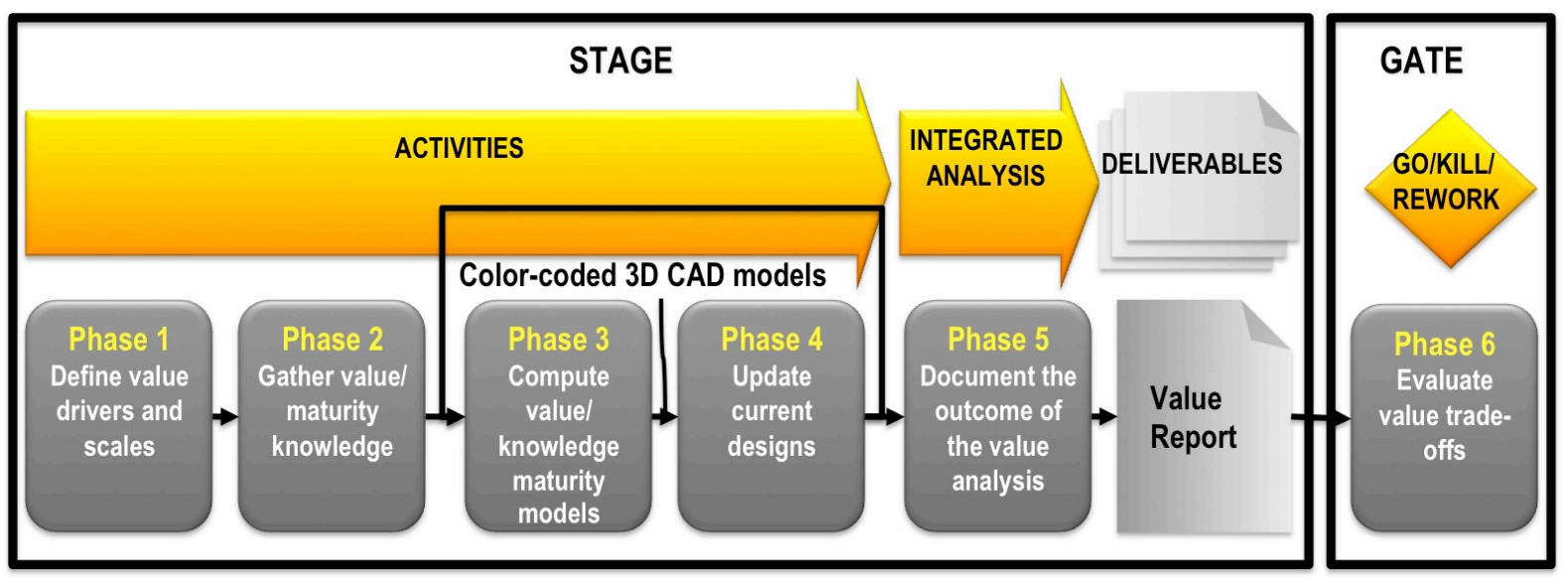

Fig. 2: The value assessment process [8]

In order to configure the value models, the design team needs to retrieve information about the relationship between drivers and objectives, for instance how MTBF affects Availability in real life (Phase 2). Furthermore, because some aspects of the product lifecycle may be more important then others, the team must assign weights to each driver. It is also important to notice that the information that characterizes such value models features different levels of confidence (some relationships might be supported by experimental evidence, others might be just educated guesses). In parallel, designers develop handful solution concepts, using as input the available list of requirements and value drivers, which are formalized within the CAD/PLM environment.

During Phase 3 the value models are fed with value drivers and objectives, and computed to produce a ranking of the current designs, highlighting negatively impacted areas and suggesting necessary corrective actions (for a more detailed description of the value model adopted in the case study see: [7]). These results are communicated to the designers, who update the designs considering both the requirement description and the value information (Phase 4). If a value dimension is below the acceptance criteria, it is discussed within the team to implement the necessary corrective actions, such as modifying the product geometry, introducing a new material or involving external resources to support the development work. The designs are then re-assessed and updated. The process is iterated until a satisfactory value is found. The number of iterations depends on the complexity of the product and on time constraints.

In the Integrated Analysis step (Phase 5), the team compiles all the material needed at the gate. The final value models are computed and included in a Value Report, which also includes feedback about the level of maturity/fidelity of the models used for the value computation.

At the gate meeting (Phase 6), the decision material is reviewed, a questions and answers session with the project leader is performed, and a decision is made about the continuation of the project. The discussion aims at resolving trade-offs between alternative concepts, focusing on areas where value contribution is perceived as weak. This session focuses both on the numbers (i.e., value) and on maturity of the knowledge behind the numbers. Where needed, additional value analyses are requested to verify the correctness of the value statement and to decide among the trade-offs. Eventually, the gate is opened, the expectations for the next gate are communicated to the project leader, the acceptance criteria for the next gate are decided and resources allocated.

The development of the color-coded 3D CAD models approach for value visualization targets Phase 4 and 6 of the process pictured in Fig.2. The decisions taken in these phases, in fact, result from the team members' debate around which value drivers to prioritize, which lifecycle aspects to improve, which engineering characteristics to prefer. The ability to communicate the value of a product/technology along multiple criteria is crucial to stimulate such early stage discussions and to grow a common understanding of "value provision", eventually helping the team in making a more sound assessment on the design that is best aligned with the purpose of the project. 


\subsection{The Value Assessment Model}

In Phase 3, the value associated to the IMC concept was calculated using an ad-hoc value assessment model named Early Value-Oriented design exploration with KnowlEdge maturity (EVOKE) [7]. EVOKE is derived from the Customer Oriented Design Analysis (CODA) [40] matrix, which, in turn, is an extension of Quality Function Deployment (QFD) [2]. EVOKE takes as input the existing objectives (engineering characteristics) for a design concept (e.g., shape, size, material) together with the list of value drivers (e.g., manufacturability or availability) to produce a score (Design Merit or DM) expressing the "goodness" of the design through a percentage score from 1\% to 100\%. EVOKE uses non-linear optimization type functions to map objectives to value drivers (Fig. 3). This is believed to better approximate the customer response to changes in a product attribute [3].
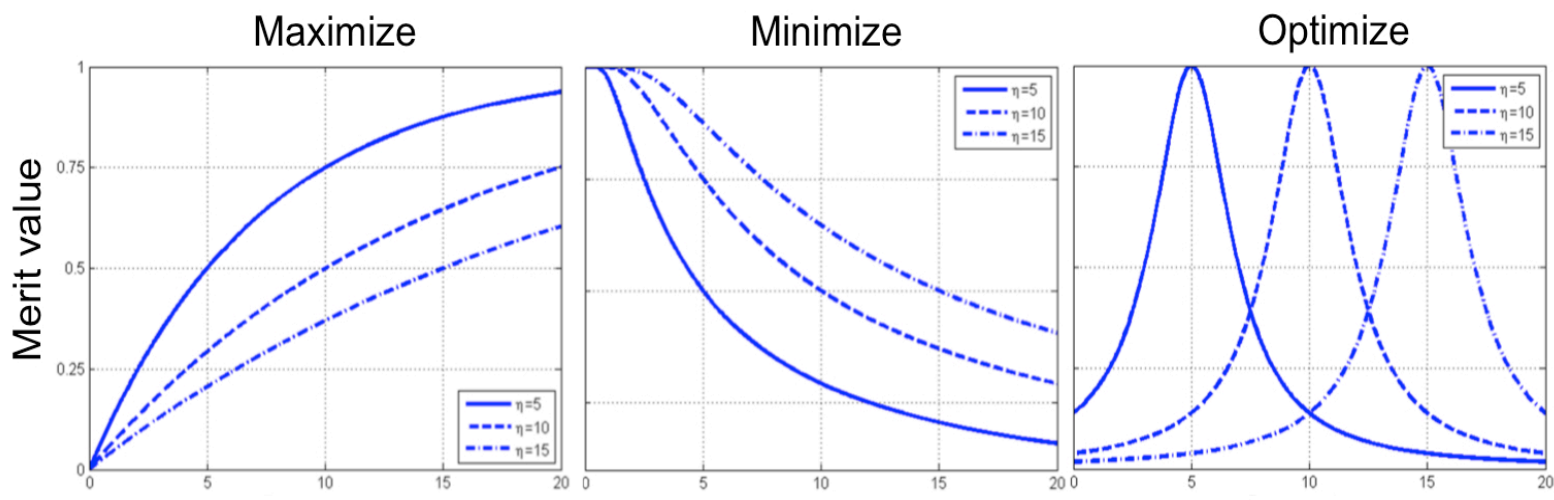

Parameter value, $\rho$

Fig. 3: Non -linear optimization type functions used in EVOKE

Table 1 shows an extract of the EVOKE matrix used to assess the IMC options in the study. Initially, the design team models the relationship between an objective and a driver using correlation coefficients. In the example, Surface finishing (the objective) is linked to Drag, Manufacturability and Knowledge Reuse (the value drivers) by strong (0.9), medium or weak correlations (0.1). A Relationship Type further characterizes this link: Drag is improved when the friction coefficient is minimized, while Manufacturability and Knowledge Reuse when is maximized (because a better surface finishing increases production lead time and requires expert workers to be executed). Neutral points and tolerances (only for "optimize" functions) allow calculating $D M$ scores for each value driver, which are then aggregated (using a normalized weight based of the criticality of each driver) to obtain the total $D M$ for the IMC.

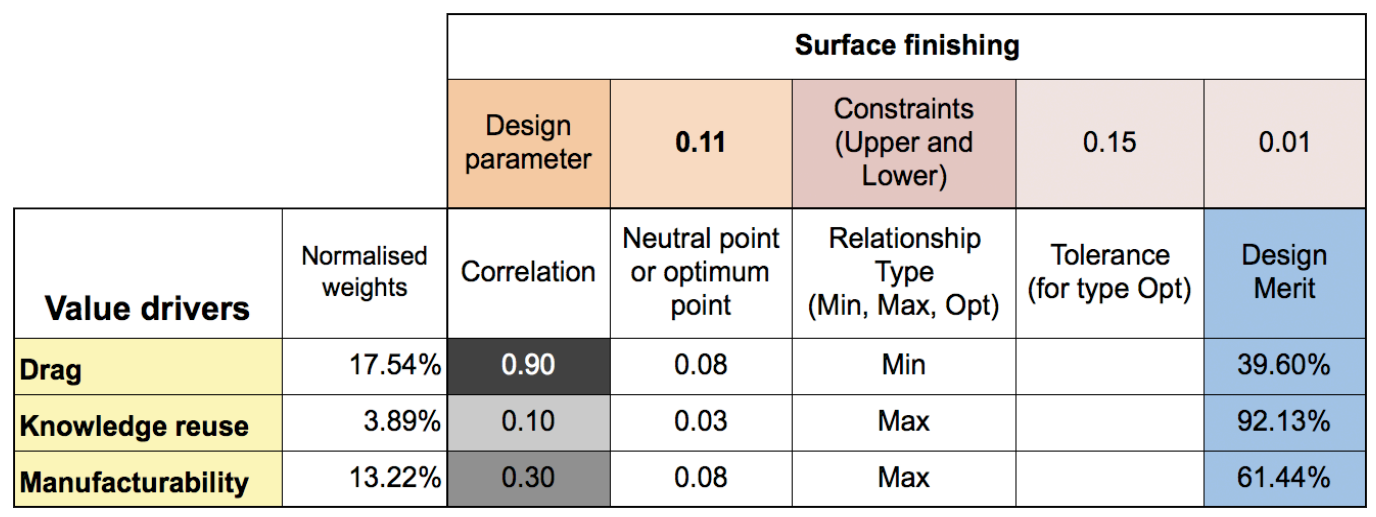

Tab. 1: Extract from the CODA matrix 


\section{PREFERENCES FOR VALUE VISUALISATION: RESULTS FROM THE EMPIRICAL STUDY}

The empirical study took place between May 2009 and October 2012. In this timeframe the authors had access to several aerospace companies, which contributed to the clarification of the problem domain, to the definition and validation of value assessment process, and to the development of the visualization approach. The authors participated to regular physical workshops, virtual work meetings and debriefing activities, where the findings were iteratively discussed and validated with the project partners. The major findings of the problem exploration phase, with regards to "what" to visualize and "how" to visualize value, are described in this section. These findings have been analyzed in the light of the available literature, and used to develop a demonstrator of the value visualization approach.

\subsection{What to Visualize?}

In spite of the centrality of the value concept in complex systems design, there is still relatively little knowledge about what value is, what its characteristics are and how stakeholders determine it. The needs analysis moved from major value-oriented methodologies in engineering design, such as Tradespace exploration [31], Value Engineering [15], Value Driven Design [12], to highlight gaps in the way value is currently visualized in Stage-Gate processes.

When using value-oriented approaches in Systems Engineering, it is important for value functions to be intuitive, meaningful, and allowing for direct comparisons among alternatives [13]. For this reason monetary units are proposed as the most convenient, practical, and universally understood metric for value [12]. However, monetizing value attributes is cumbersome and meaningless in preliminary design, and can potentially impede the timely communication of critical information between all the relevant stakeholders [37]. A preference toward using simple scalars to rank designs has clearly emerged from the study. Scalars enable direct comparisons of heterogeneous drivers, putting the focus not only on physical and functional architectures, but also on relationship-based aspects [37]. In addition, more than in producing an absolute value score, there is a preference in understanding how a concept is positioned against relevant benchmarks, to highlight if and how much a solution is better or worse than reference options. Two main references have been identified at this purpose: a product baseline (derived from historical data) and a target (which expresses a vision emerging from long-term forecasts).

A major problem with value assessment in a preliminary phase, is that value models vary a lot in terms of quality and reliability. This degree of uncertainty needs to be handled by assisting designers and decision makers in achieving a better understanding of what these uncertainties, ambiguities, and assumptions actually involve. In other words designers need to know which is the level of maturity [25] of the knowledge upon which the value models are built. The empirical study showed how critical it is to have pointers that indicate the extent to which people should trust the material entering in the value assessment activity. Models able to communicate reliability and maturity of this information have been advocated as a major enhancement to support the communication of value information.

\subsection{How to Visualize Value?}

Value is not the only criterion, but rather one of many criteria, upon which designers and process owners make decisions in a Stage-Gate process. A risk is that value contribution remains hidden by other factors, such as technical performances and cost. On the opposite side, value considerations might cause designers to lose focus on contractual requirements, causing non-conformities and delays. The empirical study has showed the need to merge heterogeneous information into a single interactive, visual environment, to facilitate decision-making activities, both in the Stage and at the Gate.

Nowadays, product and process data are presented to the user with a collection of methods, such as indented tree lists, reports and charts, which were found not to be suitable means for displaying value-related information Rather, the case study showed a preference in coupling such information with the assembly (or part) undergoing redesign. On the one hand, value-robust solutions can be more easily recognized if placed in the right context and related with the other design information. On the other hand, the implementation of 3D representations in a CAD/PLM tool allow for a streamlined navigation through the assembly/product structure, facilitating information consumption and interpretation. 
A preference has also been expressed in using multiple cues to encode value-related information in the existing project documentation, to take advantage of associative processing [33]. Multiple cues offer more opportunities for the learner to discern the new information being presented [33], and provide information quickly and automatically, decreasing time and effort needed to complete a task [5]. In this way designers can increase their perception of areas that are perceived low in terms of value contribution and make corrective actions before the gate.

The case study findings also show an inverted U-shaped relationship between the efficiency of the decision making task and the amount of value information provided, a phenomenon already observed, in a more general context, by Zahakis and his team [41]. Hence, dealing with 5 or 6 main value criteria may result to be as effective as using 200, but considerably less effective than using 25-30. In order not to overwhelm decisions makers with unnecessary details, the number of criteria to be presented at a time during the evaluation should kept to a minimum, while supporting information drilldown for access to more detailed data.

\section{TRANSLATING VALUE MODEL RESULTS INTO COLOR-CODED FEATURES IN 3D CAD MODELS}

Colors have emerged as one of the key cues for representing value-related information because of the beneficial effects for decision-making observed and discussed since the 70s. Colors are among the most effective coding technique for aiding visual search [10][29]. The processing of color precedes the processing of other attributes [26] and is highly associative [28]. Colors support learning, as they underline figure ground relationships, interrelatedness and discrimination [11]. Also, subjects with color-coded reports have been observed to obtain a significantly higher average profit in less time when performing working tasks [6].

The beneficial effects of color-coding have suggested the authors to map the value model results against a color scale, and to associate the resulting color to each relevant part/feature of the 3D CAD assembly. The aim is to create a constant link between value information and product model, and, in this way, facilitate trade-off analysis and the benchmarking of value dimensions different in nature.

As stated in section 3.1, the empirical study has shown a preference towards benchmarking alternative concepts against a baseline and target design. Hence the value model results are not directly translated into colors. Rather, the first step in the visualization consists in normalizing the outcomes of the value model using the baseline and target references. One way is to map such outcomes against a numerical scale, which express the relative value contribution of a design concept. The numerical scale chosen is a 9-point scoring system that, given a design option \#n, translates the value model result - the Design Merit $\left(D M_{n}\right)$ - into a value score $\left(S_{n}\right)$ using Eqn. (1):

$$
S_{n}=\frac{\left(S_{t}-S_{b}\right)\left(D M_{n}-D M_{b}\right)}{\left(D M_{t}-D M_{b}\right)}+S_{b}
$$

where $\left(D M_{b}\right)$ represents the Design Merit computed for the baseline and $\left(D M_{t}\right)$ for the target design. Similarly, $\left(S_{b}\right)$ represents the score for baseline, which a-priori set equal to 3, while $\left(S_{t}\right)$ represents the value score of the target, which is a-priori set equal to 8 . It has to be noted that the formula is only applicable when (Eqn. 2):

$$
\frac{\left(7 D M_{b}-2 D M_{t}\right)}{\left(S_{t}-S_{b}\right)} \leq D M_{n} \leq D M_{t}
$$

On the one hand, in case (Eqn. 3):

$$
D M_{n}>D M_{t}
$$

the algorithm automatically assigns a score of 9 to the design alternative. $S_{n}=9$ denotes a design better in value compared to what was considered as the best desirable outcome for the forthcoming solution. On the other hand, in (Eqn. 4): 


$$
D M_{n}<\frac{\left(7 D M_{b}-2 D M_{t}\right)}{\left(S_{t}-S_{b}\right)}
$$

the algorithm automatically assigns a score of 1. $S_{n}=1$ denotes a design scoring significantly below the baseline.

To exemplify the use of these formulas, let's consider a design concept that renders, as result of a value modeling activity, a $D M_{n}=65 \%$, while Baseline and Target render $D M_{b}=50 \%$ and $D M_{t}=70 \%$. Running Eqn. (1) with these numbers, the team gets a score of $S_{n}=6.75$ for the concept under analysis. In another example, the team might obtain the same result $\left(\mathrm{D} M_{n}=65 \%\right)$ from the value model, but different merits for baseline and target $\left(D M_{b}=73 \%, D M_{t}=85 \%\right)$, which render $S_{n}=0.31$. The empirical study showed, however, that the design team is not interested in knowing how much a design is lower (or higher) than existing benchmarks, but rather just to know if the value contribution of a concept falls outside the boundaries. These upper and lower boundaries are calculated using Eqn. (2). It has to be noted that, dealing with the lower boundary, the algorithm accommodates situations where the value of a design is slightly below what expressed by the baseline, to compensate for the lack of exact information when building and populating the value model. Hence, Eqn. (1) is applicable only if $D M_{n}$ is higher than $68.2 \%$ and lower than $85 \%$. Given that $D M_{n}=65 \%<68.2 \%$, Eqn. (4) assigns by default a value score $S_{n}=1$ to this concept.

The algorithm essentially renders four main areas:

- $\quad S_{n}=1 / 2$ indicates NO-GO designs, whose value contribution is below the baseline. Based on the criticality of the value driver, this may cause the design to be definitively killed for not satisfying such a minimum threshold. Otherwise, if the criticality is low, engineers may accept a lower value if this allows for more important dimensions to be improved.

- $S_{n}=3 / 4$ indicates designs that meet the minimum threshold. This score might be considered satisfactory if the criticality is low and major improvements have been achieved in dimensions with higher priority. For more critical aspects, it may trigger the decision to kill the design, especially when resources for rework activities are limited.

- $\quad S_{n}=5 / 6 / 7$ indicates designs in the GO area, although attention has to be paid on the reliability of the value assessment results. The design is moving in the right direction, but some refinements may still be made to achieve the desired value outcome.

- $\quad S_{n}=8 / 9$ indicates designs with a value equal or higher than what was originally intended. Engineers can further analyze such over-the-target dimensions to trade-off excellent capabilities with other drivers that are performing poorly, being free to decrease the value of the first in order to increase the value of the latter.

Value models are run for each part of an assembly. These parts are then color-coded according to the scores obtained from the algorithm. The use of basic colors has not been preferred, because experiments have shown that they do not segregate "exceptionally well" [36]. Rather, for specific applications, chromatic gradation within hue or color category may be more appropriate [36]. Hence, the color scale selected features different color nuances ranging from red (lowest value contribution, $S_{n}=1$ ) to green (highest value contribution, $S_{n}=9$ ). Nuances of red indicate parts where the value contribution is equal or below the baseline design. Nuances of green indicate a value contribution in line with or above the target. Nuances of orange and yellow represent areas where the value contribution is above the baseline, but not yet satisfactory for the purpose of the project.

\section{DEMONSTRATION WITH SIEMENS HD3D VISUAL REPORTING ${ }^{\circledR}$}

The value visualization approach has been prototyped and demonstrated in SIEMENS TeamCenter/NX using HD3D Visual Reporting ${ }^{\circledR}$, a visually-rich environment for working with PLM data. NX HD3D Visual Reporting provides an intuitive approach to report and conditionally format computational data in the 
3D CAD model. Its characteristics have been found particularly appealing to couple the value model results to visual features, and to foster communication and management of the value information.

The activation of NX HD3D Visual Reporting results in a conditional formatting (color-coding) of the assembly according to the particular data criteria under examination. The color-coding remains in force for as long as the report is activated, allowing the user to work on objects of interest without losing focus on the report results. Fig. 4 shows an example of color-coded IMC model for a relevant value driver (Availability). The results of the value studies conducted on each part of the assembly are first translated into colors, and then associated to the geometrical model. Aggregating the results obtained for each part, it is possible to obtain the overall value contribution of the IMC assembly (using an appropriate weighting algorithm).

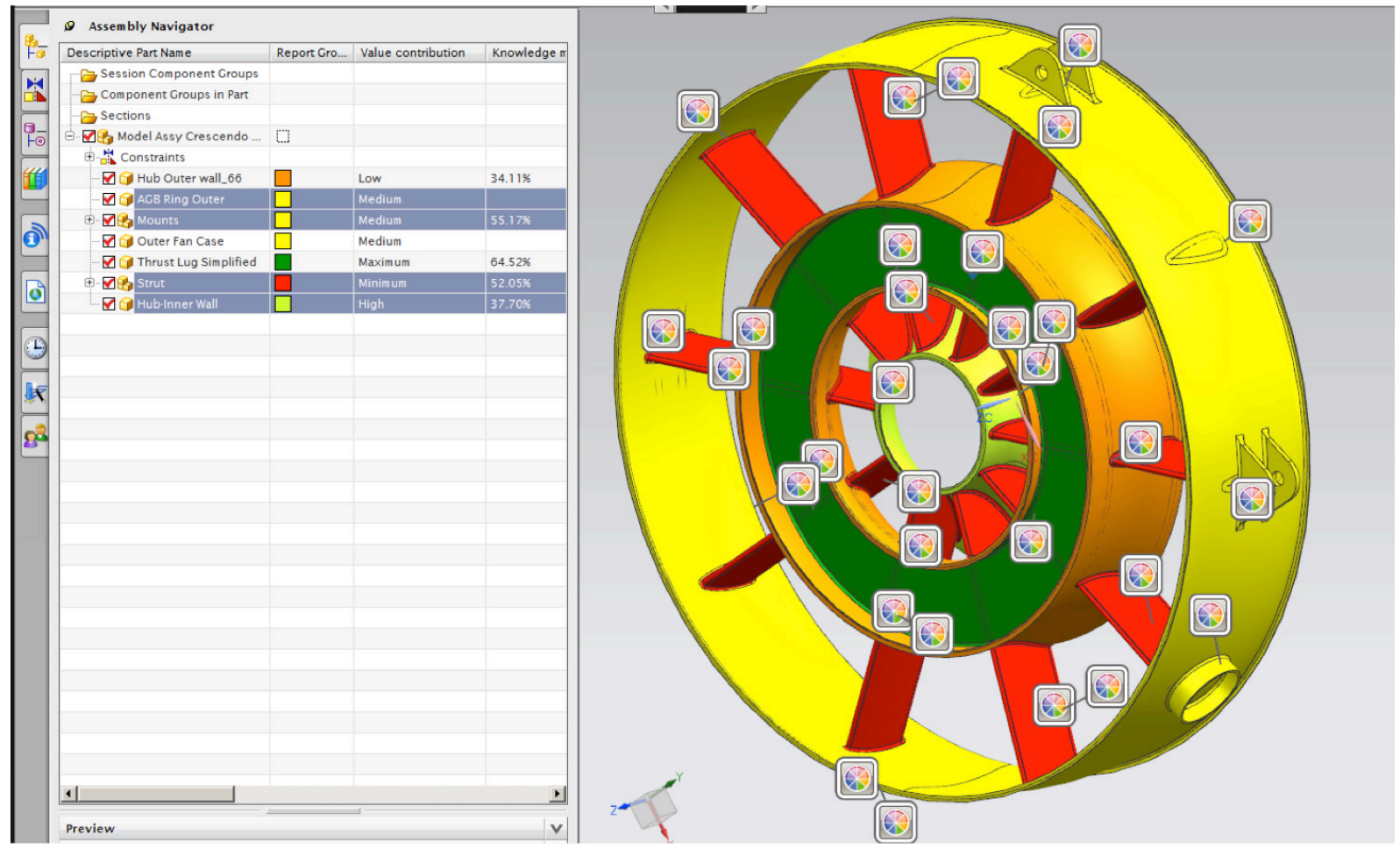

Fig. 4: Color-coded visualization of the IMC main parts (in SIEMENS NX HD3D Visual reporting).

Value Drivers and their interrelationships are managed in SIEMENS Teamcenter as requirement subtypes. Requirements are ordinary Teamcenter items individually organized in a hierarchical structure. Requirements include system defined attributes (e.g., identifier, time-stamps, user information, name, text), as well as a specific set of user defined attributes. To feed NX HD3D Visual Reporting, the value model results have been imported in an MS Excel environment and further into the SIEMENS environment. The MS Office integrations package enables to exchange data between Teamcenter and Microsoft Excel as front-end interfaces. TeamCenter offers other requirements exchange mechanisms addressing Extended Enterprise collaboration: web services APIs (SOA), PLM XML import/export, and RIF/ReqIF (Requirements Interchange Format).

NX HD3D Visual Reporting enables the users to navigate the value model results within the product structure. There is no limitation in theory to the level of granularity of the color-coded visualization, as far as the underlying value scores are present. Since value scores can be mathematically aggregated, weighted or balanced, a score, and thus the colored visualization, can characterize a part, a component, an assembly or a whole product, based on the focus of the analysis that the design team is targeting. For instance, a design team studying the design of a single aircraft engine component will most likely focus the value analysis on how the different parts (or features) 
behave in order to achieve the final design objective of that single component. A second design team, focusing of the design of a whole aircraft engine, might instead prefer a value study with less detail and that shows the value contribution of the whole IMC, without going deep into the visualization of each part. The explore report functionality offered in NX HD3D Visual Reporting lets users to choose which node level in a multi-level assembly is colored. For instance, they can display the values scores of the individual part components and their individual colors, or the overall value of the assembled components, coloring the assembly as one entity.

The legend in the Visual Reporting dialog box helps users to interpret results and perform actions on groups of components that are displayed within the same group (color). Multiple see-through settings give various methods for removing complexity from the model, displaying the components of interest. Selective focus and data rollup help users navigating up and down the product structure. Additional information and detail is accessible by drilling down on the interactive HD3D tags. This is achieved by selecting the tag and right clicking with the mouse to select InfoView. InfoView displays chosen information and opens associated documents. In the example, knowledge maturity information is associated to tags and directly attached to features of interest. By clicking on tags, users access, for instance, the description of the simulation model used to assess the value of a particular building block, as well as best practices and lessons learned reports that have been used or referenced throughout the course of the work. The bitmap displayed for the tags can be customized for added recognition and understanding. The output options allow the users to export Visual Report results to other formats for use outside the NX session.

\subsection{Color Blindness}

Working with color-coding, it is important to keep in mind that many individuals suffer from problems with recognition and understanding of colorful images and videos. Color deficiency problems are relatively common in aging population. Some estimates indicate that for the male population, approximately $7.40 \%$ of European descendents, $4.17 \%$ of Asians, and $2.61 \%$ of Africans have some kind of red-green color vision deficiency (CVD) [34].

Issues related to color deficiency are addressed by running an appropriate re-coloring algorithm. Image re-coloring is a method of altering the color composition of an image in such a way to make it accessible to a color vision impaired audience [27]. The objective of re-coloring is to preserve an abundance of visual information within the constraints of this limited color range. As mentioned by a recent paper from Culp [17], image re-coloring research counts twenty-four algorithms, broken down into four categories (color contrast enhancing; gamut re-mapping; daltonization; conversion to grayscale). Algorithms in the first three categories produce separate images for red-green and bluegreen deficiencies. Algorithms in the fourth category produce a single image [18]. Although image recoloring is not implemented in the current demonstrator, the issue has been discussed with the developers of the HD3D Visual Reporting tool. Future versions of the demonstrator will include recoloring algorithms to enable the use of the color-coding features to color-blind individuals.

\section{VERIFICATION OF THE APPROACH}

Verification activities have been conducted both in industry and academia. Interviews have been arranged with managers and designers at the case company, discussing both the value assessment process and the demonstrator. Feedback on the feasibility and relevancy of the method has been gathered by means of live presentations and webinars, involving about 30 industrial experts in each session. The approach was acknowledged to enhance decision support system capabilities, improving the mutual communication and understanding of value between the members of the cross-functional design team, and the perception of the impact on value of different design strategies. Value and knowledge maturity visualization were also acknowledged to stimulate the design team participants in probing the underlying knowledge base. Individuals have started to objectify and find facts about the system value contribution, asking questions about the underlying data quality, which otherwise would not even be considered.

The authors have also designed and conducted experiments using protocol analysis [21] and a Likert-scale questionnaire to analyze designers' behavior during design episodes. The purpose with the

Computer-Aided Design \& Applications, 10(a), 2013, bbb-ccc (c) 2013 CAD Solutions, LLC, $\underline{\mathrm{http}: / / w w w . c a d a n d a . c o m}$ 
experiments was to measure the effectiveness of color-coded CAD models as value carriers, by comparing them against a more classical form of visualization (plain CAD models complemented by color-coded tables). The experiments consisted of a design session in which 8 teams, composed of students from a Master Programme in product development, worked separately on the design of a barbeque grill. All the teams shared the same goal (to design and select a solution concept that maximizes a list of relevant value drivers) and received as input the same information, but visualized in different forms.

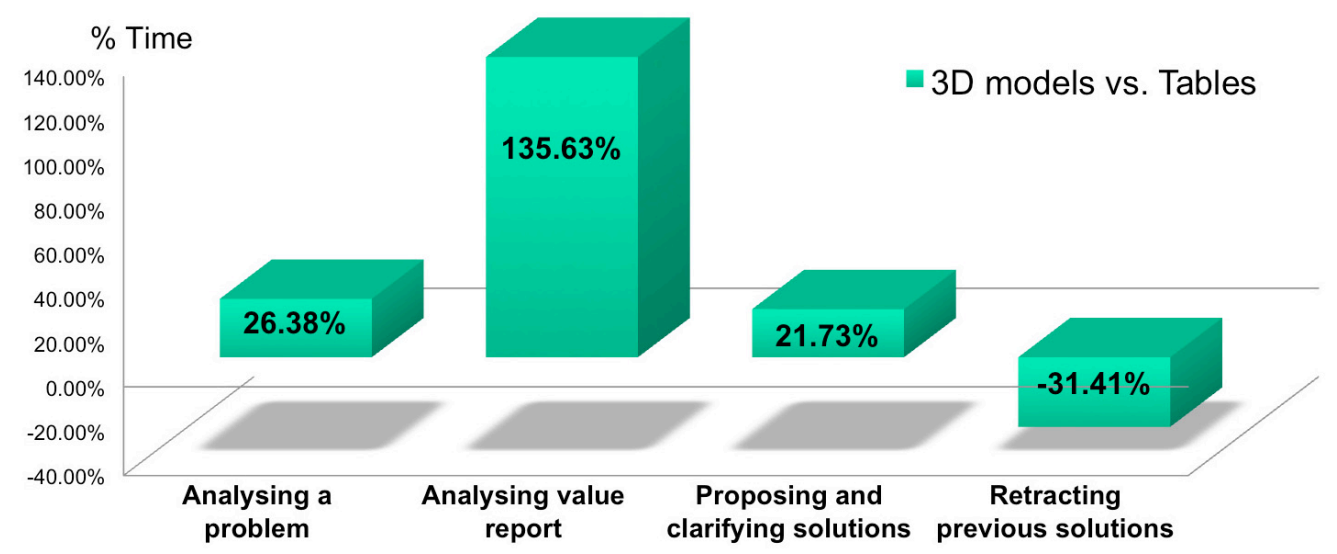

Fig. 4: Results from verification activities.

The protocol analysis results show that the 4 teams with color-coded 3D models dedicate (Fig. 4):

- $\quad 135 \%$ more time to analyze the value reports, compared to color-coded tables;

- $26 \%$ more time to analyze the design problem;

- $\quad 21 \%$ more time to propose and clarifying solutions;

- $31 \%$ less time to retract previous solutions (to identify suitable solutions in less iterations).

These results can be interpreted positively in the light of the current literature about the challenges in the early stages of product development [4][39]. Investing more time in analyzing the design problem and the related value reports would help to reduce the knowledge gap related to the "design process paradox" [39], which is the lack of information in early phases when the major part of the final product value is committed. Moreover teams able to spend more time to embed multidisciplinary aspects in their analysis, have been proven to be more successful in delivering a highly valuable solution [4]. In the individual questionnaire participants have expressed a preference toward color-coded 3D models to enhance the individual perception of the value assessment results. Their usage was acknowledged to facilitate the search for value information and to enhance the processing of the value analysis results under time pressure, while tables have been generally found more difficult to connect with the physical product. The questionnaires show also that color-coded 3D CAD models outperform tables in terms of usefulness for developing a new solution (+20\%) and awareness of the value assessment report data $(+13 \%)$.

\section{SUMMARY}

The study has highlighted a set of preferences towards the visualization of value analysis results performed during the preliminary design phase of complex systems. These preferences have guided the development of a methodological/technological approach for value visualisation, which was further implemented in a commercial CAD/PLM environment. The results of the verification activities performed so far indicate that color-coding 3D CAD models increases the decision makers' awareness of value-related information in a Stage-Gate process, stimulating the analysis of value assessment reports and the discussion on value-related matters. Such results will further be validated by means of new experiments and by coding the video and audio recording from experimental sessions. 


\section{ACKNOWLEDGMENTS}

The research leading to these results has received funding from the European Union Seventh Framework Programme (FP7/2007-2013) under grant agreement n²34344 (www.crescendo-fp7.eu/). Special thanks go to Ola Isaksson and Henrik Amnell (GKN Aerospace Engine Systems Sweden) for their support during the course of the work.

\section{REFERENCES}

[1] AIRBUS:

Taking cabin comfort to the http://www.airbus.com/en/aircraftfamilies/a350/comfort.html, AIRBUS.

[2] Akao, Y.; Mizuno, S.: QFD: The customer-driven approach to quality planning and deployment, Asian Productivity Organization, Tokio, J, 1994

[3] Anderson, E.W.; Sullivan, M.W.: The antecedents and consequences of customer satisfaction for firms, Marketing Science, 12(Spring), 1993, 125-143.

[4] Austin, S.; Steele, J.; Macmillan, S.; Kirby, P.; Spence, R.: Mapping the conceptual design activity of interdisciplinary teams, Design Studies, 22(3), 2001, 211-232.

[5] Bargh, J.A.: The four horsemen of automaticity: Awareness, intention, efficiency, and control in social cognition, in: R.S. Wyer and T.K. Srull (Eds.) Handbook of social cognition, Lawrence Erlbaum Associates Inc., Hillsdale, NJ, 1994, 1-40.

[6] Benbasat, B.: An experimental program investigating color-enhanced and graphical information presentation: an integration of the findings, Communications of the ACM archive, 29(11), 1986, 1094-1105.

[7] Bertoni, M., Bertoni, A.; Isaksson, O.; Amnell, H.; Johansson, C.: Value-oriented concept selection in aero-engine sub-systems design: the EVOKE approach, in Proc. of the $23^{\text {rd }}$ Annual INCOSE International Symposium, Philadelphia, PA, 2013, June 24-27.

[8] Bertoni, M.; Johansson, C.; Bertoni, A.: Towards assessing the value of aerospace components: a conceptual scenario, in Proc. of the $18^{\text {th }}$ International Conference on Engineering Design, Copenhagen, Denmark, 2011, 226-235.

[9] Boeing: Technology Redefines Joy of http://www.boeingcapital.com/p2p/archive/06.2006/techredefinesjoy.htm, Boeing.

[10] Christ, R.E.: Review and analysis of colour-coding research for visual displays, Human Factors, 17(6), 1975, 542-570.

[11] Chute, A.G.: Analysis of the instructional functions of color and monochrome cuing in media presentations, Educational Communication and Technology Journal, 27(4), 1979, 251-263.

[12] Collopy, P.D.; Hollingsworth, P.M.: Value-Driven Design, Journal of Aircraft, 48(3), 2011, 749-759.

[13] Collopy, P. 2012, A Research Agenda for the Coming Renaissance in Systems Engineering, American Institute of Aeronautics and Astronautics Symposium, Reston, VA, 2012, paper 20120799

[14] Cooper, R.G.: Perspective: The Stage-Gate ${ }^{\circledR}$ Idea-to-Launch Process-Update. What's New, and NexGen Systems, Journal of Product Innovation Management, 25, 2008, 213-232.

[15] Cooper R.; Slagmulder R.: Target Costing and Value Engineering, Productivity Press, Portland, OR, 1999.

[16] CRESCENDO consortium partners: D2.2.4 Validation of the system to link expectations to technical requirements, www.crescendo-fp7.eu, EU FP7 CRESCENDO project public deliverable, 2012.

[17] Culp, G.M.: Increasing Accessibility for Map Readers with Acquired and Inherited Colour Vision Deficiencies: A Re-Colouring Algorithm for Maps, The Cartographic Journal, 49(4) 2012, 302-311.

[18] Dassault Systemes, http://www.3ds.com/plm/

[19] Dieter, G.E.: Engineering design: a materials and processing approach, McGraw-Hill Inc., Boston, MA, 2000.

[20] Engelbrektsson, P.; Söderman, M.: The use and perception of methods and product representations in product development: A survey of Swedish industry, Journal of Engineering Design, 15(2), 2004, 141-154.

[21] Gero, J.S.; McNeill, T.: An approach to the analysis of design protocols, Design Studies, 19, 1998, 21-61. 
[22] Hannah, R.; Joshi, S.; Summers, J.D.: A user study of interpretability of engineering design representations, Journal of Engineering Design, 23 (6), 2012, 443-468.

[23] Hara, T.; Arai, T.; Shimomura, Y.: A CAD system for service innovation: integrated representation of function, service activity, and product behaviour, Journal of Engineering Design, 20(4), 2009, 367-388.

[24] INCOSE: Systems Engineering Handbook: A Guide for System Life Cycle Processes and Activities. Version 3.2. Revised by M. Krueger, D. Walden, and R. D. Hamelin. San Diego, CA, 2010.

[25] Johansson, C.; Hicks, B.; Larsson, A.; Bertoni, M.: Knowledge maturity as a means to support decision making during product-service systems development projects in the aerospace sector, Project Management Journal, 42(2), 2011, 32-50

[26] Karayanidis, F.; Michie, P.T.: Evidence of visual processing negativity with attention to orientation and colour in central space, Electroencephalography and Clinical Neurophysiology, 103(2), 1997, 282-297.

[27] Kuhn, G.R.; Oliveira, M.M.; Fernandes, L.A.F.: An Efficient Naturalness-Preserving ImageRecoloring Method for Dichromats, IEEE transactions on visualization and computer graphics, 14(6), 2008, 1747-1754.

[28] McNab, A.L.; Hess, T.J.; Valacich, J.S.; Designing Interfaces for Faster Information Processing: Examination of the Effectiveness of Using Multiple Information Cues, in Proc. of AMCIS' 09, San Francisco, CA, 2009

[29] Murch, G.M.: Physiological principles for the effective use of colour, IEEE Computer Graphics and Applications, 4(11), 1984, 49-54.

[30] Provost M.J.: The more electric aero- engine: A general overview from an engine manufacturer. In International Conference on Power Electronics, Machines and Drives, in Proc. of the International Conference on Power Electronics, Machines and Drives, Bath, UK, 2003, April 16-18, 246- 251.

[31] Ross, A.; Hastings, D.; Warmkessel, J.; Diller, N.: Multi-Attribute Tradespace Exploration as Front End for Effective Space System Design, Journal of Spacecraft and Rockets, 41(1), 2004, 20-28.

[32] Sedlmair, M.; Isenberg, P.; Baur, D.; Butz, A.: Information visualization evaluation in large companies: Challenges, experiences and recommendations, Information Visualization, 10(3), 2011, 248-266.

[33] Severin, W.: Another look at cue summation, Education Technololgy Research and Development, 15(3), 1967, 233-245.

[34] Sharpe, L.T.; Stockman, A.; Jägle, H.; Nathans, J.: Opsin genes, cone photopigments, color vision, and color blindness Color Vision: From Genes to Perception, in K.R., Gegenfurtner; L.T. Sharpe: Color vision: From genes to perception, ed.. Cambridge University Press, 1999, 3-51

[35] SIEMENS TeamCenter, http://www.plm.automation.siemens.com/en_us/products/teamcenter/.

[36] Smallman, H.S.; Boynton, R.M.: On the usefulness of basic color-coding in information display, Displayes, 14(3), 1993, 158-165.

[37] Soban, D.S.; Hollingsworth, P.; Price, M.E.: Defining a Research Agenda in Value Driven Design: Questions That Need to be Asked, Air Transport and Operations Symposium, 2011, 11p.

[38] Srinivasan, V.: An integration framework for product lifecycle management, Computer-Aided Design, 43(5), 2011, 464-478.

[39] Ullman, D.G.: The mechanical design process, McGraw-Hill, New York, 2003.

[40] Woolley, M.; Scanlan, J.P.; Eveson, W.: Optimising the development of a medical device using formal engineering design techniques and the coda-system, in Proc. of the 7th International Conference on Concurrent Engineering, Bremen, D, 201, June 26-29.

[41] Zanakis, S.H.; Solomon, A.; Wishart, N.; Dublish, S.: Multi-attribute decision making: a simulation comparison of select methods, European Journal of Operational Research, 107, 1998, 507-529. 